\title{
Lagrange's Equations for Rocket-Type Variable Mass Systems
}

\author{
A. Nanjangud ${ }^{1}$, F. O. Eke $^{2}$
}

\begin{abstract}
This paper presents a derivation of the equations of motion of variable mass systems based on Lagrange's equations. The derivation makes use of the control volume concept and exploits Reynolds Transport Theorem to generate equations that are reasonably compact, yet general enough to capture the dynamical behavior of variable mass systems of any shape and configuration. The only restriction is that the system should include a solid base. The equations are thus very well suited for the study of the translational as well as rotational motions of rockets and similar systems. Copyright (C) 2012 Praise Worthy Prize S.r.l. - All rights reserved.
\end{abstract}

Keywords: Dynamics, Rockets, Variable Mass Systems

\section{Nomenclature}

Inertial acceleration of a generic particle $P$ of the variable mass system

$\mathbf{a}_{\mathrm{r}} \quad$ Acceleration ofparticle $P$ relative to body $R$ or $B$

$B \quad$ A rigid massless shell that totally encloses the system and is rigidly attached to body $R$

F Resultant external force on the variable mass system

$G \quad$ Fluid portion of variable mass system

I Central inertia dyadic of $B$ and its contents

I Central inertia dyadic of matter within $B$ at time $t$

M Total moment of external forces about mass center

Mass of system

n Outward pointing normal to surface of shell $B$

$O \quad$ Arbitrary point fixed on rigid part of system

$P \quad$ A generic point of the variable mass system

p Position vector; see Fig. 1 for definition

$q_{r} \quad$ Generalized coordinates of the system

$Q_{r} \quad$ Generalized forces

$\mathbf{r}, \mathbf{r}$ Position vectors; see Fig. 1 for definition

$R \quad$ Solid part of variable mass system

$S^{*} \quad$ Mass center of contents of $B$

$T \quad$ Kinetic energy of the system

$v \quad$ Inertial velocity of $P$

$v_{r} \quad$ Velocity of $P$ relative to body $R$ or $B$

$\omega \quad$ Inertial angular velocity of body $R$ or $B$

$\alpha$

\section{Introduction}

Studies of variable mass systems have spanned almost three centuries; however, these studies can be chronologically identified to have occurred in three distinct periods. The first period went from the early $18^{\text {th }}$ century to the pre-Second World War era.

According to Grattan-Guiness [1], Bernoulli [2] was the first to explore the concept of systems with varying

mass when he studied the jet propelled hydro-reactive ship. Notable contributors after him in this period were Moore [3], von Buquoy [4], Poisson [5], Tait and Steele [6], and Meshcherskii [7]. During this first period, the focus was on the translational dynamics of systems with varying mass.

The second period of study of variable mass systems began during the Second World War and continued to the late 1970's. In this period, there was a surge of interest in rocketry with the advent of ballistic missiles. By this time, the literature on the translational motion of variable mass systems was well understood and the focus was shifted to the attitude motion. Rosser [8], Gantmacher and Levin [9], Rankin [10], Ellis and MacArthur [11] and Leitman [12] were some of the contributors in this period. A major contributor in this period was Thomson. His book [13] and companion articles [14] - [15] are some of the most significant additions to the state of knowledge of the dynamics of variable mass systems. Towards the end of this period, in 1970, Meirovitch[16] added another dimension to these studies by performing introductory work on the dynamics of flexible rockets.

The modern era of study of variable mass systems began in the 1980's, and was triggered by an anomaly observed on an upper stage powered by a then new and very large rocket. The system was operating in a spinstabilized mode and displayed an abnormal coning behavior during the rocket burn. A flurry of investigations into this anomalous behavior led to a number of new and interesting results. Some of the major studies were carried out by Flandro [17], Mingori [18], Wang [19] - [22], T.C. Mao [23] - [24], and W. Mao $[25,26]$. The main lesson learned in this period is that a spinning rocket system can be destabilized by internal phenomena that are not normally accounted for by the basic equations of motion of the system.

Practically all the results presented in the above references are for axisymmetric systems. The ultimate goal of the work that is begun in this paper is to extend 
the most recent work on the dynamics of variable mass systems to systems that are not quite axisymmetric. Since studies of this kind are usually begun by the derivation of equations that govern the motion of the system of interest, this paper presents a complete derivation of the equations of motion of a general variable mass system containing a solid base. This derivation utilizes Lagrange's equations, and exploits the control volume concept that is commonly used in fluid dynamics.

\section{The System}

Fig. 1 below is a schematic representation of the type of variable mass system that is of interest in this study. The system consists of a solid base, $R$, that is assumed to be rigid, anda fluid phase $G$.Both $R$ and $G$ are enclosed by a rigid but porous container, $B$, which is rigidly attached to $R$. It is assumed that matter can flow into, or leave the system through $B$, and that Bmaintains the sameshape at all times and has a constant volume $V$.Though it is possible for parts of $R$ to be eaten away and converted to $\mathrm{G}$, it will be assumed that some part of the system will always remain solid. As the system gains and/or loses mass with time, our interest lies only in the motion of $B$ and its contents at any given time.

To further characterize the system, a right-handed set of mutually perpendicular unit vectors, $\mathbf{b}_{1}, \mathbf{b}_{2}, \mathbf{b}_{3}$, is attached to the solid base. $O$ is any point of $R$; the vector $\mathbf{r}$ connects $O$ to a generic point $P$ of the system; $\mathbf{r}^{*}$ is the position vector from $O$ to the mass center, $S^{*}$, of the entire system, and the vector $\mathbf{p}$ connects $S^{*}$ to $P$.

Two systems $S$ and $S^{\prime}$ which are related to Fig. 1 are now defined. The system $S$ is exactly that shown in Fig. 1 and consists of $B$ and whatever is found inside $B$ at the instant of interest. It is thus a system whose mass varies with time, but whose volume remains constant at $V$ at all times. This system will be referred to, from now on, as the variable mass system $S$ associated with $B$. Now, at some general instant of time, $t$, a definite set of particles is enveloped by $B$. One can choose to focus attention on this exact set of particles, and examine its behavior, even at times different from $t$. In that case, the mass of this new system will always be the same, though the region it occupies at different times will be different, becoming the same as $B$ only at time $t$. This set of particles, that is totally enveloped by $B$ at the time $t$, will be referred to, in what follows, as the constant mass system, $S$ ', associated with $B$ at time $t$. As already stated, the mass of this system remains constant, but its volume $V$, though equal to $V$ at time $t$, will be different from $V$ at other times.

\section{Equations of Motion}

At time $t, S$ and $S^{\prime}$ ' are identical. Since $S^{\prime}$ is made up of a clearly identifiable set of particles of constant mass, its dynamic behavior can be captured by any of the equations of classical dynamics. For example, Lagrange's equations can be written for $S$ 'for the instant $t$ as:

$$
\frac{d}{d t}\left(\frac{\partial T}{\partial \dot{q}_{r}}\right)-\frac{\partial T}{\partial q_{r}}=Q_{r} \quad(r=1,2, \ldots, n)(1)
$$

where $T$ is the kinetic energy of $S^{\prime}, q_{r}$ are the generalized coordinates used to describe the configuration of $S^{\prime}, Q_{r}$ are the generalized forces and $n$ is the number of degrees of freedom of $S^{\prime}$.

We will now pause, and develop the kinematic quantities that will be needed to generate expressions for each of the terms that appear in (1).

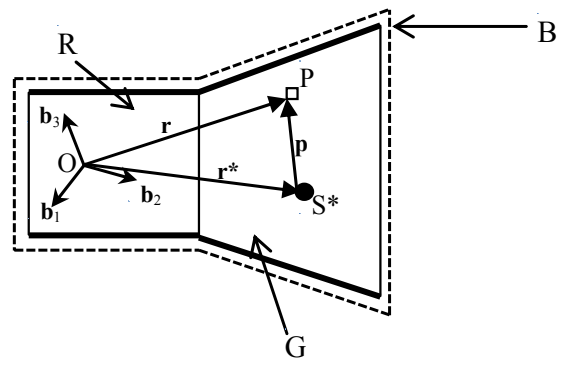

Fig. 1. Variable mass system with solid base

\section{III.1. Kinematics}

The constant mass system $S$ 'described above has an infinite number of degrees of freedom. To reduce the potential complexity of the problem of deriving equations of motion for this system, it is assumed that both the configuration and motion of the fluid particles of $G$, relative to the solid phase, are known at all times.

This reduces the number of degrees of freedom of the system $S$ 'to six.Let the generalized coordinates used to describe the configuration of $S$ be $q_{1}, q_{2}, \ldots, q_{6}$; where $q_{1}$, $q_{2}, q_{3}$ are Cartesian coordinates of some point $O$ of $B$ relative to an inertial reference frame $N$, and $q_{4}, q_{5}, q_{6}$ are orientation angles (e.g. Euler angles) that specify the orientation of $R$ or $B$ in $N$.

The inertial velocity of point $O$ can be written as:

$$
\mathbf{v}^{O}=u_{1} \mathbf{b}_{1}+u_{2} \mathbf{b}_{2}+u_{3} \mathbf{b}_{3}
$$

where:

$$
u_{i}=f_{i}\left(\dot{q}_{l}, q_{j}, t\right) \text { with }(i=1,2,3 \& j=4,5,6)
$$

Similarly, the inertial angular velocity of $B$ is defined to be:

$$
\omega=u_{4} \mathbf{b}_{1}+u_{5} \mathbf{b}_{2}+u_{6} \mathbf{b}_{3}
$$

where $u_{j}=f_{j}\left(q_{j}, q_{j}, t\right)$ and $(j=4,5,6)$. For points $P$ and $S^{*}$, the velocity expressions are, respectively:

$$
\mathbf{v}=\mathbf{v}^{O}+\omega \times \mathbf{r}+\mathbf{v}_{r}
$$

and: 


$$
\mathbf{v}^{*}=\mathbf{v}^{O}+\omega \times \mathbf{r}^{*}+\mathbf{v}_{r}^{*}
$$

In (4) and (5), $\mathbf{v}_{\mathrm{r}}$ and $\mathbf{v}_{r}^{*}$ are, respectively, the velocities of $P$ and $S^{*}$ relative to the body $B$. If a subscript $q_{r}$ is used to denote partial derivative with respect to $q_{r}$, then (4) and (5) lead to:

$$
\begin{gathered}
\frac{\partial \mathbf{v}}{\partial q_{r}}=\mathbf{v}_{q_{r}}=\mathbf{v}_{q_{r}}^{o}+\left(\boldsymbol{\omega}_{q_{r}} \times \mathbf{r}\right) \quad \mathbf{r}=1,2, \ldots, 6 \\
\mathbf{v}_{q_{r}}^{*}=\mathbf{v}_{q_{r}}^{o}+\left(\boldsymbol{\omega}_{q_{r}} \times \mathbf{r}^{*}\right) \quad \mathbf{r}=1,2, \ldots, 6
\end{gathered}
$$

The corresponding partial derivatives with respect to $\dot{q}_{r}$ would then be:

$$
\mathbf{v}_{\dot{q}_{r}}=\mathbf{v}_{\dot{q}_{r}}^{o}+\left(\omega_{\dot{q}_{r}} \times \mathbf{r}\right) \quad \mathbf{r}=1,2, \ldots, 6
$$

and:

$$
\mathbf{v}_{\dot{q}_{r}}^{*}=\mathbf{v}_{\dot{q}_{r}}^{o}+\left(\omega_{\dot{q}_{r}} \times \mathbf{r}^{*}\right) \quad \mathbf{r}=1,2, \ldots, 6
$$

Subtracting (5) from (4) gives:

$$
\mathbf{v}=\mathbf{v}^{*}+\omega \times \mathbf{p}+\left(\mathbf{v}_{r}-\mathbf{v}_{r}^{*}\right)
$$

Similarly, (6) and (7) yield:

$$
\mathbf{v}_{q_{r}}=\mathbf{v}_{q_{r}}^{*}+\left(\boldsymbol{\omega}_{q_{r}} \times \mathbf{p}\right) \quad \mathbf{r}=1,2, \ldots, 6
$$

and (8) and (9) give:

$$
\mathbf{v}_{\dot{q}_{r}}=\mathbf{v}_{\dot{q}_{r}}^{*}+\left(\omega_{\dot{q}_{r}} \times \mathbf{p}\right) \quad \mathbf{r}=1,2, \ldots, 6
$$

Next, the time derivatives, in the inertial reference frame, of the quantities $\mathbf{v}_{\dot{q}_{r}}^{*}$ and $\mathbf{v}_{q_{r}}$ are evaluated:

$$
\frac{d}{d t}\left(\mathbf{v}_{\dot{q}_{r}}\right)=\frac{d}{d t}\left(\frac{\partial \mathbf{v}}{\partial \dot{q}_{r}}\right)=\frac{\partial}{\partial \dot{q}_{r}}\left(\frac{d \mathbf{v}}{d t}\right)
$$

Since $\mathbf{v}=\mathbf{v}\left(\dot{q}_{i}, q_{j}, t\right), i=1,2, \ldots, 6 ; j=4,5,6$ :

$$
\frac{d v}{d t}=\sum_{i=1}^{6} \frac{\partial v}{\partial \dot{q}_{i}} \ddot{q}_{i}+\sum_{j=4}^{6} \frac{\partial v}{\partial q_{j}} \dot{q}_{j}+\frac{\partial v}{\partial t}
$$

and hence:

$$
\begin{aligned}
& \frac{\partial}{\partial \dot{q}_{r}}\left(\frac{d \mathbf{v}}{d t}\right)=\frac{\partial \mathbf{v}}{\partial q_{r}} \\
& \text { or } \frac{d \mathbf{v}_{\dot{q}_{r}}}{d t}= \begin{cases}0 & r=1,2,3 \\
\mathbf{v}_{q_{r}} & r=4,5,6\end{cases}
\end{aligned}
$$

Copyright $(02012$ Praise Worthy Prize S.r.l. - All rights reserved
Similarly, it is easy to show that:

$$
\frac{d \omega_{\dot{q}_{r}}}{d t}= \begin{cases}0 & r=1,2,3 \\ \omega_{q_{r}} & r=4,5,6\end{cases}
$$

\section{III.2. Kinetic Energy}

The kinetic energy, $T$, of the system $S$ ' is given by:

$$
T=\frac{1}{2} \int_{V^{\prime}} \rho \mathbf{v} \cdot \mathbf{v} d V
$$

Hence, from (17) and (11):

$$
\frac{\partial T}{\partial q_{r}}=\int_{V^{\prime}} \rho\left[\mathbf{v}_{q_{r}}^{*} \cdot \mathbf{v}+\boldsymbol{\omega}_{q_{r}} \cdot(\mathbf{p} \times \mathbf{v})\right] d V
$$

and, from (17) and (12):

$$
\frac{\partial T}{\partial \dot{q}_{r}}=\int_{V^{\prime}} \rho\left[\mathbf{v}_{\dot{q}_{r}}^{*} \cdot \mathbf{v}+\omega_{\dot{q}_{r}} \cdot(\mathbf{p} \times \mathbf{v})\right] d V
$$

Taking the time derivative of (19) and substituting (15) and (16) into the outcome leads to:

$$
\frac{d}{d t}\left(\frac{\partial T}{\partial \dot{q}_{r}}\right)=\int_{V^{\prime}} \rho\left[\begin{array}{l}
\mathbf{v}_{q_{r}}^{*} \cdot \mathbf{v}+\mathbf{v}_{\dot{q}_{r}}^{*} \cdot \mathbf{a}+\omega_{q_{r}} \cdot(\mathbf{p} \times \mathbf{v})+ \\
+\omega_{\dot{q}_{r}} \cdot \frac{d}{d t}(\mathbf{p} \times \mathbf{v})
\end{array}\right] d V
$$

where $\mathbf{a}$ is the acceleration of point $P$ in an inertial reference frame. At this point, expressions have been determined for the terms on the left hand side of (1). On the right hand side of (1) are the generalized forces $Q_{r}$.

These can be expressed as:

$$
Q_{r}=\int_{V^{\prime}} \mathrm{d} \mathbf{F} \cdot \mathbf{v}_{\dot{q}_{r}} d V
$$

where $\mathbf{F}$ is the resultant force on the generic element $P$ of $S^{\prime}$. Replacing $\mathbf{v}_{\dot{q}_{r}}$ with its expression from (12), one obtains:

$$
\begin{aligned}
Q_{r} & =\int_{V^{\prime}}\left[\mathrm{d} \mathbf{F} \cdot \mathbf{v}_{\dot{q}_{r}}^{*}+\boldsymbol{\omega}_{\dot{q}_{r}} \cdot(\mathbf{p} \times \mathrm{d} \mathbf{F})\right] d V= \\
& =\mathbf{v}_{\dot{q}_{r}}^{*} \cdot \mathbf{F}+\boldsymbol{\omega}_{\dot{q}_{r}} \cdot \mathbf{M}
\end{aligned}
$$

In (22), $\mathbf{F}$ is the resultant external force acting on the entire system S', and imagined applied at the mass center, and $\mathbf{M}$ is the sum of the moments of all the external forces acting on the system about the system mass center.

\section{III.3. Dynamical Equations}

The dynamical equations for the system $S$ 'can now be assembled by substituting (20), (18), and (22) into (1). 
However, this substitution is done in two phases once for values of the subscript $r$ from 1 to 3 , and then for values of $r$ ranging from 4 to 6.For $r=1,2,3$, (1) reduces to:

$$
\int_{V^{\prime}} \rho \mathbf{v}_{\dot{q}_{r}}^{*} \cdot \mathbf{a} d V=\mathbf{v}_{\dot{q}_{r}}^{*} \cdot\left(m \mathbf{a}^{*}\right)=\mathbf{v}_{\dot{q}_{r}}^{*} \cdot \mathbf{F}
$$

where $m$ is the mass of $S^{\prime}$ and $\mathbf{a}^{*}$ is the inertial acceleration of the mass center of $S^{\prime}$. For $r=4,5,6$, (1) becomes:

$$
\int_{V^{\prime}} \rho\left[\mathbf{v}_{\dot{q}_{r}}^{*} \cdot \mathbf{a}+\boldsymbol{\omega}_{\dot{q}_{r}} \cdot \frac{d}{d t}(\mathbf{p} \times \mathbf{v})\right] d V=\mathbf{v}_{\dot{q}_{r}}^{*} \cdot \mathbf{F}+\boldsymbol{\omega}_{\dot{q}_{r}} \cdot \mathbf{M}
$$

Because $\mathbf{v}_{\dot{q}_{r}}^{*}$ is not zero in general, nor is it generally perpendicular to $\mathbf{F}$ or $\mathbf{a}^{*}$, the scalar equation (23) can be replaced with the vector expression:

$$
m \mathbf{a}^{*}=\mathbf{F}
$$

Equation (24) can be re-written as:

$$
\begin{aligned}
& \mathbf{v}_{\dot{q}_{r}}^{*} \cdot m \mathbf{a}^{*}+ \\
& \quad+\boldsymbol{\omega}_{\dot{q}_{r}} \cdot \int_{V^{\prime}} \rho \frac{d}{d t}(\mathbf{p} \times \mathbf{v}) d V=\mathbf{v}_{\dot{q}_{r}}^{*} \cdot \mathbf{F}+\boldsymbol{\omega}_{\dot{q}_{r}} \cdot \mathbf{M}
\end{aligned}
$$

Using (25) in (26) leads eventually to the simple vector equation:

$$
\int_{V^{\prime}} \rho \frac{d}{d t}(\mathbf{p} \times \mathbf{v}) d V=\frac{d}{d t} \int_{V^{\prime}} \rho(\mathbf{p} \times \mathbf{v}) d V=\mathbf{M}
$$

Equations (25) and (27) can be said to describe the motion of the constant mass system $S^{\prime}$. However, our real interest is in the equations of motion of the variable mass system $S$.

We now return to (25), and recall that $\mathbf{a}^{*}$ is not the acceleration of the mass center of the variable mass system $S$, but rather, that of the mass center of the constant mass system $S^{*}$. Clearly, this acceleration is next to impossible to determine for a real system. We now re-write (25) as:

$$
\begin{aligned}
& m\left[\mathbf{a}^{O}+\boldsymbol{\alpha} \times \mathbf{r}^{*}+\boldsymbol{\omega} \times\left(\boldsymbol{\omega} \times \mathbf{r}^{*}\right)+2 \boldsymbol{\omega} \times \mathbf{v}_{r}^{*}+\mathbf{a}_{r}^{*}\right]= \\
& =m\left[\mathbf{a}^{O}+\boldsymbol{\alpha} \times \mathbf{r}^{*}+\boldsymbol{\omega} \times\left(\boldsymbol{\omega} \times \mathbf{r}^{*}\right)\right]+ \\
& +2 \int_{V^{\prime}} \rho\left(\boldsymbol{\omega} \times \mathbf{v}_{r}\right) d V+\int_{V^{\prime}} \rho \mathbf{a}_{r} d V=\mathbf{F}
\end{aligned}
$$

where $\mathbf{a}_{r}$ is the acceleration of $P$ relative to $B$. The last term on the left hand side of (28) can be re-written in such a way that it can be expanded by the Reynolds Transport Equation [27]. Thus,

$$
\begin{aligned}
\int_{V^{\prime}} \rho \mathbf{a}_{r} d V & =\int_{V^{\prime}} \rho \frac{{ }^{B} d \mathbf{v}_{r}}{d t} d V=\frac{{ }^{B} d}{d t} \int_{V^{\prime}} \rho \mathbf{v}_{r} d V= \\
& =\frac{{ }^{B} d}{d t} \int_{V} \rho \mathbf{v}_{r} d V+\int_{S} \rho \mathbf{v}_{r}\left(\mathbf{v}_{r} \cdot \mathbf{n}\right) d S
\end{aligned}
$$

Here, $B$ is taken to be a control region of volume $V$ and surface area $S ; \mathbf{n}$ is an outward pointing unit normal to $B$, and the left superscript $B$ is used to indicate that the time derivative is taken in the $B$ reference frame (noninertial frame). Substituting (29) into (28) yields:

$$
\begin{aligned}
& m\left[\mathbf{a}^{O}+\boldsymbol{\alpha} \times \mathbf{r}^{*}+\boldsymbol{\omega} \times\left(\boldsymbol{\omega} \times \mathbf{r}^{*}\right)\right]+ \\
& \quad+2 \int_{V^{\prime}} \rho\left(\boldsymbol{\omega} \times \mathbf{v}_{r}\right) d V+\frac{{ }^{B} d}{d t} \int_{V} \rho \mathbf{v}_{r} d V+ \\
& \quad+\int_{S} \rho \mathbf{v}_{r}\left(\mathbf{v}_{r} \cdot \mathbf{n}\right) d S=\mathbf{F}
\end{aligned}
$$

At time $t$, the first term on the lefthand side of (30) is the same for both the systems $S^{\prime}$ and $S$; and $V=V^{\prime}$.

Hence (30) is valid for the system $S$ at time $t$, and represents the equation of translational motion for the variable mass system $S$ at the general instant $t$.

Next, we go back to (27), into which (10) is substituted, and then by invoking the definition of mass center, the resulting expression is:

$$
\frac{d}{d t} \int_{V^{\prime}} \rho\left[\begin{array}{l}
\mathbf{p} \times(\omega \times \mathbf{p})+ \\
+\mathbf{p} \times \mathbf{v}_{r}
\end{array}\right] d V=\mathbf{M}
$$

If the reference frame in which the time derivative is taken in (31) is changed from the inertial frame to the frame $B,(31)$ becomes:

$$
\begin{aligned}
& \frac{{ }^{B} d}{d t} \int_{V^{\prime}} \rho \mathbf{p} \times(\omega \times \mathbf{p}) d V+\frac{{ }^{B} d}{d t} \int_{V^{\prime}} \rho \mathbf{p} \times \mathbf{v}_{r} d V+ \\
& \quad+\omega \times \int_{V^{\prime}} \rho \mathbf{p} \times(\omega \times \mathbf{p}) d V+ \\
& \quad+\omega \times \int_{V^{\prime}} \rho \mathbf{p} \times \mathbf{v}_{r} d V=\mathbf{M}
\end{aligned}
$$

Invoking Reynolds Transport Theorem, and after some algebra, (32) takes the form:

$$
\begin{aligned}
& \frac{{ }^{B} d}{d t}(\mathbf{I} \cdot \boldsymbol{\omega})+\boldsymbol{\omega} \times \mathbf{I}^{\prime} \cdot \boldsymbol{\omega}+\int_{S} \rho \mathbf{p} \times(\boldsymbol{\omega} \times \mathbf{p})\left(\mathbf{v}_{r} \cdot \mathbf{n}\right) d S+ \\
& +\frac{{ }^{B} d}{d t} \int_{V} \rho \mathbf{p} \times \mathbf{v}_{r} d V+\int_{S} \rho \mathbf{p} \times \mathbf{v}_{r}\left(\mathbf{v}_{r} \cdot \mathbf{n}\right) d S+ \\
& +\boldsymbol{\omega} \times \int_{V^{\prime}} \rho \mathbf{p} \times \mathbf{v}_{r} d V=\mathbf{M}
\end{aligned}
$$


which can also be written as:

$$
\begin{aligned}
& \mathbf{I} \cdot \boldsymbol{\alpha}+\left(\frac{{ }^{B} d \mathbf{I}}{d t}\right) \cdot \boldsymbol{\omega}+\boldsymbol{\omega} \times \mathbf{I}^{\prime} \cdot \boldsymbol{\omega}+ \\
& +\int_{\mathbf{S}} \rho \mathbf{p} \times(\boldsymbol{\omega} \times \mathbf{p})\left(\mathbf{v}_{r} \cdot \mathbf{n}\right) d S+\frac{{ }^{B} d}{d t} \int_{V} \rho \mathbf{p} \times \mathbf{v}_{r} d V+ \\
& \quad+\int_{S} \rho \mathbf{p} \times \mathbf{v}_{r}\left(\mathbf{v}_{r} \cdot \mathbf{n}\right) d S+\boldsymbol{\omega} \times \int_{V^{\prime}} \rho \mathbf{p} \times \mathbf{v}_{r} d V=\mathbf{M}
\end{aligned}
$$

In both (33) and (34), I and I' represent thecentralinertia dyadics of $S$ and $S$ ' respectively. These quantities are equal at time $t$, but their time derivatives are different in general.

In summary, the translational motion of a general variable mass system such as the one shown in Fig. 1 is governed by equation (30) above. The attitude motion of the same system is governed by (33) or (34). The first term on the left hand side of (30) represents the rigid body contribution, and the remaining terms are due to mass variability.

In the same way, the equation of rotational motion of a variable mass system is seen to comprise rigid body terms and extra terms due to mass variability.

\section{Conclusion}

The equations of motion are derived for a general variable mass system that includes a solid base. The derivation is done by Lagrange's method and utilizing the control volume approach. Several variants of the attitude equations for such a system exist in the literature.

However, a version of the equations of motion that is deemed the mosttractable in the study of rockets has been identified by prior investigators [19] - [24] and it is this form of the attitude equation that is obtained in this paper.

\section{References}

[1] I. Grattan-Guinness and R. Cooke,Landmark Writings in Western Mathematics 1640-1940. (Elsevier, Amsterdam, 2005).

[2] D. Bernoulli, Hydrodynamica, Argentorati (1738)

[3] W. Moore, A Treatise on the Motion of Rockets, (London, 1813)

[4] G. Buquoy, Exposition d'un Nouveau Principe General de Dynamique (Paris, 1816)

[5] S. D. Poisson, Sur le Mouvement d'un System de Corps, en Supposant les Masses Variables,Bull. Sci. Soc. Philomat, , Paris (April1819), 60-62

[6] P. G. Tait and W. J. Steele, A treatise on the Dynamics of a Particle. (Cambridge, 1856)

[7] I. V. Meshcherskii, Equations of Motion of a Variable Mass Point in the General Case, St. Petersburg Polytechnic University News, 1, (1904), 77-118

[8] J. B. Rosser and R. R. Newton et al, Mathematical Theory of Rocket Flight. (McGraw-Hill, New York, 1947).

[9] F. R. Gantmacher and L. M. Levin, 'Equations of Motion of a Rocket'. NACA TM 1255(1950)

[10] R. A. Rankin, The Mathematical Theory of Rotated and Unrotated Rockets, Philosophical Transactions Royal Society London Series A 241(1949), 457-585

[11] J. W. Ellis and C. W. McArthur, Applicability of Euler's
Dynamical Equations to Rocket Motion, ARS Journal 29 (1959), 863-864

[12] G. Leitman, On the Equation of Rocket Motion, Journal of British Interplanetary Society 16(1957), 141-147

[13] W. T. Thomson, Introduction to Space Dynamics, (Dover, New York, 1986)

[14] W. T. Thomson, Jet Damping of a Solid Rocket: Theory and Flight Results, AIAA Journal, 3(3)(1965), 413-417

[15] W. T. Thomson, Equations of Motion for the Variable Mass System, AIAA Journal, 4(4)(1966),766-768

[16] L. Meirovitch, General Motion of A Variable Mass Flexible Rocket, Journal of Spacecraft and Rockets 7 (2) (1970), 186-195

[17] G. A. Flandro et al., Fluid Mechanics of Spinning Rockets, AFRPL-TR-86-072, Edwards AFB, California

[18] D. L. Mingori, and Y. Yam, Nutational Instability of a Spinning Spacecraft with Internal Mass Motion and Axial Thrust, AIAA paper 86-2271, AIAA/AAS Astrodynamics Conference, Williamsburg, VA, (1986)

[19] S. M. Wang, Attitude Dynamics of Variable Mass Systems, Ph. D. Dissertation, UC Davis, 1993

[20] S. M. Wang and F. O. Eke, Rotational Dynamics of Axisymmetric Variable Mass Systems, J. Appl. Mech. 62, (1995)

[21] F. O. Eke and S. M. Wang, Attitude Behavior of a Variable Mass Cylinder, J. Appl. Mech. 62, (1995)

[22] F. O. Eke and Song-Min Wang, Equations of Motion of TwoPhase Variable Mass Systems With Solid Base, J. Appl. Mech. 61, (1994)

[23] T. C. Mao, Attitude Motions of Space-Based Variable Mass Systems, Ph. D. Dissertation, UC Davis, 1996

[24] T. C. Mao and F. O. Eke, Attitude dynamics of a torque-free variable mass cylindrical body. The Journal of the Astronautical Sciences 48(4) (2000)435-448.

[25] W. Mao and F.O. Eke, Equations of Motion of Receiver Aircraft During In-Flight Refueling, International Review of Aerospace Engineering (IREASE), 2 (6), (2009), 321 - 328.

[26] W. Mao and F.O. Eke, Effects of Mass Variation on the Motion of Receiver Aircraft During In-Flight Refueling, International Review of Aerospace Engineering (IREASE), 5 (3),(2012), 109 116.

[27] F. M. White, Fluid Mechanics, $2^{\text {nd }}$ Edition, (McGraw-Hill,New York, 1986).

\section{Authors' information}

${ }^{1,2}$ Department of Mechanical and Aerospace Engineering, University of California, Davis, CA 95616, USA

Angadh Nanjangud has a B.E. in Mechanical Engineering from Bangalore University. He is currently a doctoral student in Mechanical and Aerospace Engineering at the University of California at Davis. His research focus is on the dynamics of variable mass systems.

F. O. Eke holds a Ph.D. in Applied Mechanics from Stanford University, Stanford, CA. He is currently Professor of Mechanical and Aerospace Engineering at the University of California, Davis, CA. His research interests are in the general areas of dynamics and control of aerospace vehicles. 